\title{
Static Irradiated ALSEP Scrub Section
}

\author{
Dean R. Peterman
}

April 2017

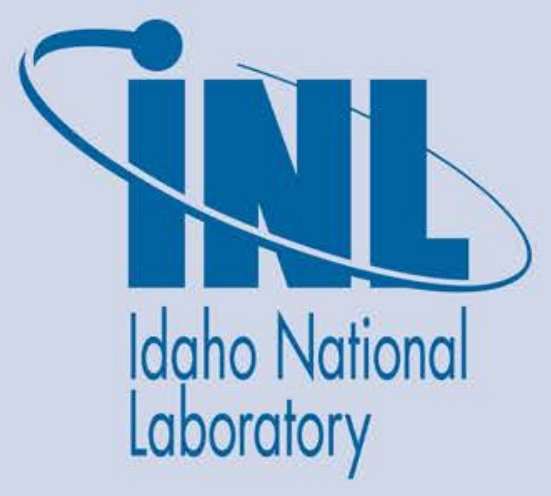

The INL is a U.S. Department of Energy National Laboratory operated by Battelle Energy Alliance 
INL/EXT-17-41644

\title{
Static Irradiated ALSEP Scrub Section
}

\author{
Dean R. Peterman
}

April 2017

\section{Idaho National Laboratory \\ Idaho Falls, Idaho 83415}

http://www.inl.gov

Prepared for the

U.S. Department of Energy

Office of Nuclear Energy

Under DOE Idaho Operations Office

Contract DE-AC07-05ID14517 


\section{Static Irradiated ALSEP Scrub Section}

The INL is studying the impacts of gamma radiolysis on the ALSEP minor actinide extraction process. This process uses an organic solvent consisting of a neutral diglycolamide extractant, $N, N, N^{\prime}, N^{\prime}$-tetra-2ethylhexylglycolamide (T2EHDGA), and an acidic extractant 2-ethylhexylphosphonic acid mono-2ethylhexyl ester (HEH[EHP]) dissolved in an aliphatic diluent. The actinides and lanthanides are coextracted from relatively high nitric acid and the actinides are selectively stripped using a carboxylic acid buffered aminopolycarboxylic acid solution. Previous investigations of the radiolytic degradation processes on the ALSEP process performed at the INL focused upon the extraction section of the flowsheet. Current efforts are concerned with examining the scrub section of the ALSEP flowsheet. This report describes the static irradiations performed during the current fiscal year in support of the test loop irradiations which will be conducted later in FY-2017, and documents completion of the level four milestone (M4FT17IN030102012) entitled "Static Irradiated ALSEP Scrub Section.

To support the ALSEP test loop irradiations planned for FY-2017, samples of the ALSEP solvent were irradiated under static conditions in the INL's ${ }^{60}$ Co self-contained gamma irradiator. All static irradiated samples were sparged with air $(1 \mathrm{sccm})$ during irradiation. The solvent composition used for all irradiations was $0.050 \mathrm{M}$ T2EHDGA + 0.75 M HEH[EHP] in n-dodecane. The organic solvent was pre-equilibrated with nitric acid or simulated ALSEP feed solution three times at an organic to aqueous phase ratio (O/A) of one. The ALSEP simulant was obtained from PNNL and adjusted to $3 \mathrm{M} \mathrm{HNO}_{3}$ prior to use. The T2EHDGA was obtained from Eichrom and was used as received. The HEH[EHP] was obtained from CarboSynth and was purified by the third phase formation process prior to use. All other reagents were obtained from Sigma Aldrich and were used as received. All radiotracers used were taken from laboratory stocks.

The irradiated aqueous and organic phases were used to perform simplified extraction, scrub, and strip batch contact flowsheet tests. The scrub solution contained $0.2 \mathrm{M}$ citric acid adjusted to $\mathrm{pH}=3$ and the strip solution contained $0.125 \mathrm{M}$ HEDTA $+0.2 \mathrm{M}$ citric acid adjusted to $\mathrm{pH}=3$. The americium, europium, and cerium distribution ratios $\left(\mathrm{D}_{\mathrm{Am}}, \mathrm{D}_{\mathrm{Eu}}\right.$, and $\left.\mathrm{D}_{\mathrm{Ce}}\right)$ determined for the batch contact flowsheet test using unirradiated ALSEP solvent and $3 \mathrm{M} \mathrm{HNO}_{3}$ are listed in Table 1.

Table 1 Distribution ratios determined for a simplified ALSEP extraction, scrub, and strip batch contact flowsheet test. Aqueous: un-irradiated $3 \mathrm{M} \mathrm{HNO}_{3}$; Organic: un-irradiated ALSEP solvent.

\begin{tabular}{c|c|c|c|} 
& $\mathrm{D}_{\mathrm{Am}}$ & $\mathrm{D}_{\mathrm{Eu}}$ & $\mathrm{D}_{\mathrm{Ce}}$ \\
\hline Extraction & 21 & 140 & 6.6 \\
Scrub A & 19 & 460 & 35 \\
Scrub B & 51 & 1100 & 100 \\
Strip & 0.48 & 27 & 15
\end{tabular}

The results presented in Table 1 serve as a baseline for comparison of the distribution ratios obtained using ALSEP solvent static irradiated in contained with a simulated ALSEP feed solution. The results of the batch contact flowsheet test performed using ALSEP solvent static irradiated in contact with a simulated feed 
solution containing $0.05 \mathrm{~mol} / \mathrm{L}$ trans-1,2-diaminocyclohexane- $N, N, N$ ' $N$ '-tetraacetic acid (CDTA) are presented in Table 2.

Table 2. Distribution ratios determined as a function of absorbed dose for a simplified ALSEP extraction, scrub, and strip batch contact flowsheet test. Aqueous: irradiated ALSEP feed containing $0.05 \mathrm{M}$ CDTA adjusted to $3 \mathrm{M} \mathrm{HNO}_{3}$; Organic: irradiated ALSEP solvent. nd: not determined.

\begin{tabular}{|c|c|c|c|c|c|c|c|c|c|}
\hline \multirow{2}{*}{$\begin{array}{c}\text { Absorbed } \\
\text { Dose, } \\
\text { kGy }\end{array}$} & \multicolumn{3}{|c|}{ Ext 1} & \multicolumn{3}{|c|}{ Scr 2} & \multicolumn{3}{|c|}{ Stp 1} \\
\hline & $\mathrm{D}_{\mathrm{Am}}$ & $\mathrm{D}_{\mathrm{Eu}}$ & $\mathrm{D}_{\mathrm{Ce}}$ & $\mathrm{D}_{\mathrm{Am}}$ & $\mathrm{D}_{\mathrm{Eu}}$ & $\mathrm{D}_{\mathrm{Ce}}$ & $\mathrm{D}_{\mathrm{Am}}$ & $\mathrm{D}_{\mathrm{Eu}}$ & $\mathrm{D}_{\mathrm{Ce}}$ \\
\hline 0 & 2.05 & 9.80 & 0.57 & 30 & 755 & 51 & 0.27 & 21 & 10 \\
\hline 23 & 1.80 & 8.45 & 0.55 & 28 & 793 & 56 & 0.28 & 18 & 9 \\
\hline 47 & 1.62 & 7.93 & 0.51 & 29 & 573 & 53 & 0.27 & 17 & 9 \\
\hline 93 & 1.40 & 6.13 & nd & 30 & 781 & nd & 0.29 & 17 & nd \\
\hline 186 & 1.25 & 5.26 & 0.43 & 27 & 559 & 47 & 0.30 & 17 & 10 \\
\hline 278 & 1.01 & 3.96 & 0.35 & 21 & 596 & 45 & 0.29 & 11 & 8 \\
\hline 395 & 0.80 & 3.10 & 0.29 & 29 & 589 & 46 & 0.33 & 11 & 10 \\
\hline 465 & 0.68 & 2.45 & 0.25 & 29 & 700 & 54 & 0.38 & 12 & 10 \\
\hline 511 & 0.63 & 2.12 & 0.25 & 29 & 360 & 55 & 0.43 & 12 & 12 \\
\hline
\end{tabular}

When compared with the distribution ratios determined for the extraction of americium, europium, and cerium from nitric acid (see Table 1), the distribution ratios determined using the simulated ALSEP feed solution at zero absorbed dose are suppressed. This decrease in the measured distribution ratios is expected due to the partial loading of the ALSEP solvent by the lanthanides and other fission products present in the simulated feed.

The decrease in the magnitude of the extraction $\mathrm{D}_{\mathrm{Am}}, \mathrm{D}_{\mathrm{Eu}}, \mathrm{D}_{\mathrm{Ce}}$ values as a function of increasing absorbed dose (shown in Table 2) demonstrates that the ALSEP is susceptible to radiolytic degradation. Radiolytic degradation of the ALSEP solvent is, also, indicated by the observed increase of the magnitude of the values of the americium stripping distribution ratios as a function of increased absorbed dose. However, it should be noted the impact of radiolytic degradation is not severe and no removal of the products of gamma radiolytic degradation was attempted.

Based upon the results obtained from the static irradiation experiments, three ALSEP test loop irradiation experiments will be performed in FY-2017:

1. Irradiation of ALSEP solvent in contact with $3.0 \mathrm{M} \mathrm{HNO}_{3}$.

2. Irradiation of ALSEP solvent in contact with simulated ALSEP feed containing CDTA adjusted to 3.0 $\mathrm{M} \mathrm{HNO}_{3}$.

3. Irradiation of loaded ALSEP solvent in contact with ALSEP scrub solution.

Samples from each of these test loop irradiations will be collected as a function of absorbed dose. These samples will be used to determine the impact of gamma radiolysis upon the ALSEP separation by determining values of metal distribution ratios as a function of absorbed dose using radiotracer techniques. The distribution of stable metal species present in the ALSEP will be determined by ICP-MS analysis. In addition, the composition of the ALSEP solvent will be determined using analytical techniques developed or optimized at the INL. 